\title{
BMJ Open Prediction and prognostication of neurological deterioration in patients with acute ICH: a hospital-based cohort study
}

\author{
Christian Ovesen, ${ }^{1}$ Anders Fogh Christensen, ${ }^{2}$ Inger Havsteen, ${ }^{2}$ \\ Christine Krarup Hansen, ${ }^{1}$ Sverre Rosenbaum, ${ }^{1}$ Engin Kurt, ${ }^{2}$ Hanne Christensen ${ }^{1}$
}

To cite: Ovesen $C$, Christensen AF, Havsteen I, et al. Prediction and prognostication of neurological deterioration in patients with acute ICH: a hospital-based cohort study. BMJ Open 2015;5:e008563. doi:10.1136/bmjopen-2015008563

- Prepublication history and additional material is available. To view please visit the journal (http://dx.doi.org/ 10.1136/bmjopen-2015008563).

Received 21 April 2015 Revised 2 July 2015 Accepted 6 July 2015

CrossMark

\footnotetext{
${ }^{1}$ Section of Acute Neurology Departments of Neurology, Bispebjerg Hospital, Copenhagen, Denmark ${ }^{2}$ Department of Radiology, Bispebjerg Hospital, Copenhagen, Denmark
}

Correspondence to Christian Ovesen; christian.aavang.ovesen@ regionh.dk

\section{ABSTRACT}

Objective: Patients with intracerebral haemorrhage (ICH) are at high risk of neurological deterioration (ND). We aimed at establishing predictors of early ND (END) as well as late ND (LND) and at exploring the impact of neurological stability during the first week on long-term prognosis.

Design: We conducted this study as a retrospective cohort study. ND was evaluated based on the consciousness and severity of neurological symptoms. ND during the first $24 \mathrm{~h}$ after admission was defined as early ND and from $24 \mathrm{~h}$ to 7 days as LND. Patients were followed up until February 2015.

Participants: We included 300 patients with acute $\mathrm{ICH}$ ( $\leq 4.5 \mathrm{~h}$ from symptom onset) who were admitted to our institution from March 2009 to January 2015.

Setting: Section of Acute Neurology, Department of Neurology, Bispebjerg Hospital is a specialised referral centre receiving patients with acute stroke from the entire capital region of Denmark.

Results: We found that a spot sign on CT angiography (OR $10.7 \mathrm{Cl} 4.79$ to 24.3) and extensive degree of interventricular haemorrhage (IVH) (OR 8.73 $\mathrm{Cl} 2.87$ to 26.5) were independent predictors of END, whereas a degree of comorbidity (Charlton Index), admission stroke severity and degree of IVH predicted LND. On follow-up imaging, haematoma expansion was independently associated with END (OR $6.1 \mathrm{Cl} 2.2$ to 17.3), and expansion of IVH was independently associated with both END (OR $1.7 \mathrm{Cl} 1.2$ to 2.3 per point increase) and LND (OR $2.3 \mathrm{Cl} 1.3$ to 4.2 per point increase). ND during the first week was associated with a 1-year mortality of $60.5 \%$, compared with $9.2 \%$ among the patients who remained stable.

Conclusions: These results suggest that stability during the first week entails an optimistic prognosis. A relatively easy and effective risk stratification of END and LND is possible on admission based on the spot sign, IVH and clinical parameters.

\section{INTRODUCTION}

Neurological deterioration (ND) in patients with intracerebral haemorrhage (ICH) is

\section{Strengths and limitations of this study}

- The main strengths of this study are its fairly large sample size of patients with acute intracerebral haemorrhage (ICH) included consequently without selection.

- The large proportion of the patients worked up with CT angiography in the acute setting allowing us to include the spot sign as a prognostic marker; and the detailed follow-up regarding outcome allowing very few people to be lost to follow-up.

- The major limitation of this study is the incomplete use of follow-up imaging $24 \mathrm{~h}$ after stroke onset. Both patients with mild $\mathrm{ICH}$ and those with devastating strokes might be less likely to receive follow-up imaging. We chose to include all patients to keep the study population as unselected as possible.

common, as approximately $25 \%$ of patients deteriorate within the first 2 days following admission. ${ }^{1-5}$ However, even though the incidence of early ND (END) is well described, easy-to-use predictors allowing a better ultraearly risk stratification in terms of identifying unstable patients are not well established. New imaging concepts such as the CT angiography (CTA)-based spot sign, which have been proved to predict haematoma expansion, are most likely a powerful tool in the acute risk stratification of patients with ICH. Further, little is known about late ND (LND), even though the existence of LND is well established among clinicians. ${ }^{4} \quad 6$ Evidence indicates that structural damage as well as secondary space-occupying lesions in the brain occurs predominantly during the very first days of illness ${ }^{7-12}$ (provoking END and LND). Assuming minimisation of complications by means of adequate stroke unit care (including aspiration pneumonia, 
venous embolisms, urinary tract infections, etc), ${ }^{13} 14$ we propose that clinical neurological stability during the initial week after stroke onset will translate into an overall stability of the patient and hence a lower risk of long-term morbidity and mortality. Therefore, the aim of the present study was to establish predictors of END and LND present on admission as well as the significance of the acute phase of illness on the long-term prognosis.

\section{METHOD}

The Department of Neurology, Bispebjerg Hospital maintains a database with ongoing registration of consecutive patients with primary ICH admitted to the acute stroke unit within $4.5 \mathrm{~h}$ after symptom onset. This study was conducted as a retrospectively planned analysis based on this cohort including patients admitted to our acute stroke unit from March 2009 to January 2015. The number of arriving patients determined the sample size. Patients were excluded if deeply comatose on admission (Glasgow Coma Scale (GCS) <5) or if later diagnostic workup revealed an underlying cause of the haemorrhage (final diagnosis not primary ICH). On arrival, patients underwent a standardised workup including non-contrast CT (NCCT), and a National Institute of Health Stroke Scale (NIHSS) score was obtained. Patients further underwent acute CTA if no contraindication to the procedure (allergy or significant kidney failure) was present. Patients with ICH were treated in accordance with guidelines from the European Stroke Organisation. ${ }^{15} 16$ They were continuously monitored, and vital values as well as GCS and neurological symptoms (stroke in progression (SIP) score) were recorded at least every hour within the first $24 \mathrm{~h}$ by the nursing staff. The SIP score is a shortened version of the Scandinavian Stroke Scale and is used for purposes of monitoring patients with acute stroke ${ }^{17}$ (see online supplementary materials). Follow-up imaging was scheduled to be performed approximately $24 \mathrm{~h}$ after admission or earlier in case of ND. When patients were assumed to be stable, they were transferred to rehabilitation in local hospital stroke units based on the abode of the patient. Local stroke units are monitored on quality, and all comply with the national standards regarding stroke unit care. A more detailed description of treatment procedures is presented in the online supplementary materials.

\section{Neurological deterioration}

ND was defined as a decrease of $\geq 2$ GCS points ${ }^{18}$ or $\geq 4$ SIP score points, lasting longer than $8 \mathrm{~h}$, requiring surgical intervention, or resulting in death. The cut point of 4 SIP score points was made based on the widely accepted 4 point NIHSS cut point ${ }^{319}$ when defining ND and the conversion formula between the Scandinavian Stroke Scale and NIHSS. ${ }^{20}$ END was defined as occurring within the first $24 \mathrm{~h}$ after admission, ${ }^{3} 181921$ with the admission value of GCS and SIP score as the baseline reference. The presence of END was confirmed based on the documented GCS and SIP score values along with medical chart descriptions. LND was defined as occurring later than $24 \mathrm{~h}$ after hospital admission, but within the first 7 days. ${ }^{6}$ LND was confirmed based on a review of all medical chart data from days 1 to 7 . Documented progression in the patient's condition equal to $\geq 2$ GCS points or $\geq 4$ SIP score points with the GCS and SIP score values at $24 \mathrm{~h}$ after admission as reference and with no other obvious non-neurological cause such as infection or pulmonary embolism was defined as LND. Only patients who were stable during the first $24 \mathrm{~h}$ and non-surgically treated were assessed for LND. If patients were discharged within the first week after symptom onset, they were presumed to be continuously stable. A consultant neurologist (HC) with extensive experience in stroke neurology assessed the medical charts to confirm LND.

\section{Definitions of patient characteristics and comorbidity}

Detailed descriptions on the definition of patient characteristics are presented in online supplementary materials. Disability prior to stroke onset was graded using the modified Rankin Scale (mRS), and degree of comorbidity was graded using the Charlson Comorbidity Index. $^{22}$

\section{CT and CTA imaging and analysis}

A senior consultant neuroradiologist (AFC) and two board-certified radiologists (IH and EK) reviewed all imaging in a systematic manner blinded to clinical data. We defined the presence of the spot sign as one or more 1-2 $\mathrm{mm}$ foci of enhancement within the haematoma on CTA source images ${ }^{23}$ (imaging protocol details are presented in the online supplementary materials). Haematoma volumes were calculated using the $\mathrm{ABC}$ / 2-method. ${ }^{24}$ Significant haematoma expansion was defined as an increase in haematoma volume of $\geq 12.5 \mathrm{~mL}$ between admission and follow-up NCCT. The volume and extent of the interventricular haemorrhage (IVH) were graded using the semiquantitative Graeb Score. ${ }^{25}$ Subarachnoid extension of the haemorrhage (SAH) was defined as clearly visible blood in the subarachnoid space. Delayed IVH or SAH was defined as IVH or SAH on follow-up imaging, not present on admission imaging. Midline shift was measured as the distance from the septum pellucidum between the frontal horns of the lateral ventricles to a line connecting the anterior and posterior insertions of the falx cerebri on a CT slice containing the third ventricle. ${ }^{8}$ Hydrocephalus was defined as marked dilation of one or more of the ventricles.

\section{Follow-up}

Patients were followed through the national electronic chart system. Outcome at 90 days was assessed based on records from the outpatient clinic or occupational 
therapy. Good outcome was defined as the ability to walk independently $(\mathrm{mRS} \leq 3)$. Mortality of the patients was collected during the first year after the admission date or until 20 February 2015. Patients who were not Danish citizens were censored the day they left the country.

\section{Statistics}

We compared normally distributed data using Student $\mathrm{t}$ test, non-normally distributed data and ordinal scale data using the Mann-Whitney U-test and categorical data using the $\chi^{2}$ test. We designed prediction models for END and LND using logistic regression. Baseline data that were significantly different between the two groups $(\mathrm{p}<0.05)$ were added into the models and excluded in a backward stepwise manner. In the models, we included NIHSS as the estimator of admission neurological status due to its widespread international use. GCS or SIP score was not additionally included due to collinearity concerns. On the basis of data from the follow-up imaging, we constructed two additional logistic models for END and LND. Radiological variables significantly different between the groups were included in the models. If measures of enlargement of radiological variables (eg, absolute haematoma expansion) were used in the models, we also adjusted for baseline variables (eg, admission haematoma volume). Non-adjusted survival analysis during the first year after admission was undertaken using Kaplan-Meier curves stratified for ND during the first 7 days. ND was used as a collective term for END and LND. The independent effect of ND on 90 days functional outcome and long-term mortality was assessed using logistic regression (good 90-day outcome) and Cox Proportional Hazard Model (long-term mortality). We included age and gender and degree of comorbidity (Charlson Score), factors known to be associated with ICH outcome, ${ }^{26}$ and surgical procedures in the models. ND was entered as a time-dependent variable to avoid the immortal time bias. The $\mathrm{p}<0.05$ was considered to be significant. Statistical analyses were performed in SPSS V.20 statistical software (IBM Corp, Armonk, New York, USA).

\section{RESULTS}

We included 300 patients with acute primary ICH in the cohort (figure 1). All patients received admission NCCT (224 (74.7\%) underwent acute CTA) with a median (IQR) delay from symptom onset of $103.0 \mathrm{~min}$ (76.3143.0). At least one follow-up NCCT was performed in $163(54.3 \%)$ patients with a median (IQR) delay from admission CT of 19 (10-25) h. ${ }^{10-25}$ One patient underwent external ventricular drain installation immediately after admission without indication of END. This patient was excluded from further analysis. Five patients underwent surgical evacuation of the haematoma, and three underwent placement of external ventricular drainage without previous ND. These patients were excluded from analysis at the time they underwent surgery.

\section{Early neurological deterioration}

Of the 299 patients, $89(29.7 \%)$ fulfilled the criteria of END (figure 1). Among admission characteristics (see online supplementary table S1), END was most notably associated with higher NIHSS (17 (11-22) vs 10 (5-16), $\mathrm{p}<0.0001)$, lower GCS (12 (10-14) vs $15 \quad(13-15)$, $\mathrm{p}<0.0001)$, higher admission haematoma volume $(45.1 \mathrm{~mL}$ $(18.0-88.9)$ vs $10.1 \mathrm{~mL}(3.5-24.0), \mathrm{p}<0.0001)$, and larger degree of midline shift $(2.0 \mathrm{~mm}(0.0-7.0)$ vs $0.0 \mathrm{~mm}(0.0$ $0.0), \mathrm{p}<0.0001)$, in addition to a higher frequency of IVH (51.7\% vs $24.8 \%, \mathrm{p}<0.0001)$, SAH $(37.1 \%$ vs $16.7 \%$, $\mathrm{p}<0.0001)$ and spot sign $(73.4 \%$ vs $18.1 \%, \mathrm{p}<0.0001)$. END was significantly associated with prestroke oral

Figure 1 Study outline and prevalence of neurological deterioration during the first week. Panel $(A)$ describes the incidence of END during the first $24 \mathrm{~h}$, and panel $(\mathrm{B})$ describes the incidence of LND during the following 6 days (CTA, CT angiography; END, early neurological deterioration; GCS, Glasgow Coma Scale; ICH, intracerebral haemorrhage; LND, late neurological deterioration; NC-CT, non-contrast CT). 
anticoagulation treatment $(22.7 \%$ vs $9.5 \%, \mathrm{p}=0.005)$ but not treatment with platelet inhibitors. Duration of time from admission to reversal of anticoagulation treatment commenced was similar for stable patients and patients suffering END. Of the patients with END, 20 (22.5\%) required intubation with a median (IQR) time from symptom onset of $3.0 \mathrm{~h}$ (2.0-4.0). Eleven (12.4\%) patients with END underwent placement of an external ventricular drain with a median (IQR) time to drain placement of $6.0 \mathrm{~h}(4.0-17.0)$. Seventeen (19.1\%) of the patients with END underwent surgical removal of the ICH-all with the indication of pending herniation. Of the patients with END, $34(38.2 \%)$ had a do-not-resuscitate (DNR) order placed on their charts within the first $24 \mathrm{~h}$ after admission compared with only $11(5.2 \%)$ of the stable patients $(\mathrm{p}<0.0001)$. In multivariate analysis among the 224 patients with CTA on admission (table 1), the most notable predictors of END were the spot sign, anticoagulation treatment, and extensive IVH. This model provided excellent discriminative capability towards END (C-statistics=0.87, CI 0.82 to 0.92). If CTA data were not considered $(\mathrm{n}=299)$, the significant predictors of END were oral anticoagulation treatment, extensive $\mathrm{IVH}$, and more severe stroke symptoms (NIHSS) on admission. Patients with haematoma volumes below $10 \mathrm{~mL}$ were at low risk. This model also provided a good discriminative capability towards END (C-statistics $=0.82$, CI 0.77 to 0.88 ). On a follow-up scan, significant haematoma expansion and IVH expansion were independently associated with END (table 2). Of the 36 patients with END who were alive and non-surgically treated after the initial $24 \mathrm{~h}, 19(52.8 \%)$ deteriorated further within the following 6 days.

\section{Late neurological deterioration}

After the first $24 \mathrm{~h}, 204$ patients remained stable and non-surgically treated. During the next 6 days, $20(9.6 \%)$ patients who were stable during the initial $24 \mathrm{~h}$ suffered LND (figure 1). Patients with LND (see online supplementary table S1) most notably had a significantly higher degree of comorbidity (median Charlson Score 1 $(0.5-2)$ vs $0(0-1), \mathrm{p}=0.003)$, higher admission NIHSS (15 (13-20) vs 9 (5-15), $\mathrm{p}<0.0001)$, lower admission GCS (13 (11-14) vs 15 (13-15), p=0.002), higher admission volume (28.5 mL (12.5-44.1) vs $9.2 \mathrm{~mL}(3.0-21.1)$, $\mathrm{p}=0.002)$, and higher frequency of IVH $(60.0 \%$ vs $19.6 \%, \mathrm{p}<0.0001)$ compared with patients who remained stable. In multivariate analysis, the significant predictors present on admission of LND were higher degree of comorbidity (Charlson Score), more extensive IVH, and higher admission NIHSS. This model provided excellent discriminative capability towards LND (C-statistics $=0.86$, CI 0.76 to 0.95 ). On a follow-up scan, final extension of the IVH was found to be associated with LND, and expansion of the IVH was an independent predictor when adjusted for extension of baseline IVH (table 2). Of the patients with LND, $12(60.0 \%)$ had a DNR order placed on their charts with a median (IQR) delay of 3 days (0-4) after admission. Of the patients who
Table 1 Independent predictors of END and LND

OR $95 \% \mathrm{Cl} \quad$ p Value

END-patients with CTA on admission $(n=224)$

Anticoagulation treatment $3.41 \quad 1.19$ to 9.80

0.023

Admission Graeb Score

0 points

1-5 points

1.00

6-12 points

$2.02 \quad 0.76$ to 5.40

0.161

Admission haematoma volume

$>60 \mathrm{~mL}$

$10-59.9 \mathrm{~mL}$

1.00

$<10 \mathrm{~mL}$

$0.43 \quad 0.18$ to 1.06

$0.27 \quad 0.09$ to 0.82

Spot sign

$10.7 \quad 4.79$ to 24.3

$<0.001$

END—all patients $(n=299)$

Anticoagulation treatment

Admission Graeb Score

0 points

1-5 points

$3.87 \quad 1.65$ to 9.11

0.002

6-12 points

Admission SAH

1.00

1.59

4.63

$-$

0.73 to 3.46

1.79 to 12.0

$2.24 \quad 1.03$ to 4.87

0.241

0.002

Admission haematoma volume

$>60 \mathrm{~mL}$

10-59.9 mL

1.00

$<10 \mathrm{~mL}$

0.44

0.20

$-$

0.20 to 0.95

0.07 to 0.56

Admission NIHSS (per

1.08

1.03 to 1.14

0.066

0.021

$<0.001$

point)

LND-all patients at risk at day $1(n=204)$

Charlson Score (per point)

Haematoma location

Lobar

$1.74 \quad 1.18$ to 2.55

0.005

Basal ganglia

1.00

0.17

$-$

Posterior fossa

0.005

Admission Graeb Score

0 points

1-5 points

1

6-12 points

Admission NIHSS (per

point)

Variables offered to both END models were anticoagulation treatment, admission Graeb Score, SAH on admission, serum glucose level, admission midline shift, admission haematoma volume, and admission NIHSS value (the spot sign presents only the first model). Variables offered to the LND model were

Charlson Score, admission NIHSS, admission haematoma volume, admission Graeb Score, and admission midline shift. CTA, CT angiography; END, early neurological deterioration; LND, late neurological deterioration; NIHSS, National Institute of Health Stroke Scale; SAH, subarachnoid hemorrhage.

remained stable during the entire first week, only 7 (3.7\%) had a DNR order placed on their charts with a median delay (IQR) from admission of 0 days $(0-0)$.

\section{Outcome}

After 90 days, $122(72.2 \%)$ of the surviving patients, who remained stable during the first 7 days, were able to walk independently compared with $9(20.5 \%)$ of the patients who suffered ND $(p<0.0001)$. Adjusted for significant prognostic factors (age, admission NIHSS, IVH, admission haemorrhage volume, and haematoma location), 
Table 2 Follow-up imaging characteristics

\begin{tabular}{|c|c|c|c|c|}
\hline & \multicolumn{2}{|c|}{ Early neurological deterioration } & \multicolumn{2}{|c|}{ Late neurological deterioration } \\
\hline & Crude OR & Adjusted OR* & Crude OR & Adjusted OR† \\
\hline Significant haematoma expansion, $>12.5 \mathrm{~mL}$ & $15.0(6.3$ to 35.5$)$ & $6.1(2.2$ to 17.3$)$ & $2.0(0.4$ to 10.5$)$ & $\mathrm{NI}$ \\
\hline Follow-up Graeb Score, per point & $1.3(1.2$ to 1.5$)$ & $\mathrm{NI}$ & $1.5(1.2$ to 1.9$)$ & $\mathrm{NI}$ \\
\hline Delayed IVH & 10.3 (3.2 to 33.3$)$ & $\mathrm{NI}$ & $2.6(0.2$ to 26.6$)$ & $\mathrm{NI}$ \\
\hline IVH expansion, per Graeb point & 11.0 (4.4 to 27.2$)$ & 1.7 (1.2 to 2.3$)$ & 5.5 (1.1 to 26.3$)$ & 2.3 (1.3 to 4.2$)$ \\
\hline $\mathrm{SAH}$ on the follow-up scan & 3.4 (1.6 to 7.3$)$ & 2.8 (0.8 to 9.3$)$ & $2.6(0.7$ to 9.5$)$ & $\mathrm{NI}$ \\
\hline Delayed SAH & $3.4(0.6$ to 21.3$)$ & $\mathrm{NI}$ & NA & NA \\
\hline Hydrocephalus & $5.3(1.7$ to 16.3$)$ & $1.4(0.3$ to 6.6$)$ & $2.6(0.2$ to 26.6$)$ & $\mathrm{NI}$ \\
\hline Midline shift, per $\mathrm{mm}$ & $1.3(1.1$ to 1.4$)$ & 1.1 (0.9 to 1.3$)$ & $1.2(0.9$ to 1.4$)$ & $\mathrm{NI}$ \\
\hline
\end{tabular}

comorbidity (Charlson Score) and surgical procedures (surgical evacuation and instalment of external ventricular drainage), ND was an important inverse predictor of good outcome (OR 0.11 CI 0.04 to 0.32 ) (see online supplementary table S2).

One year after admission, $60.5 \%$ (CI $51.2 \%$ to $69.8 \%$ ) of the patients who suffered ND during the first 7 days after admission had died (figure 2A). In comparison, only $9.2 \%$ (CI $4.9 \%$ to $13.5 \%$ ) of the patients who remained stable during the first 7 days had died $(\mathrm{p}<0.0001)$. The median survival of the patients who suffered ND was 20 days. Patients with END and LND suffered comparable mortality during the first year (figure 2B). When the same significant prognostic factors as above were controlled for, ND was established as an important predictor of long-term mortality (HR 6.99 CI 3.54 to 13.8) (see online supplementary table S2). Other independent predictors of mortality during the first year were age, Charlson Score, admission NIHSS, posterior fossa location, and admission haematoma volume. We only included surgical treatment of patients with pending herniation, and it appeared to affect survival; however, it was not a predictor of good functional outcome.
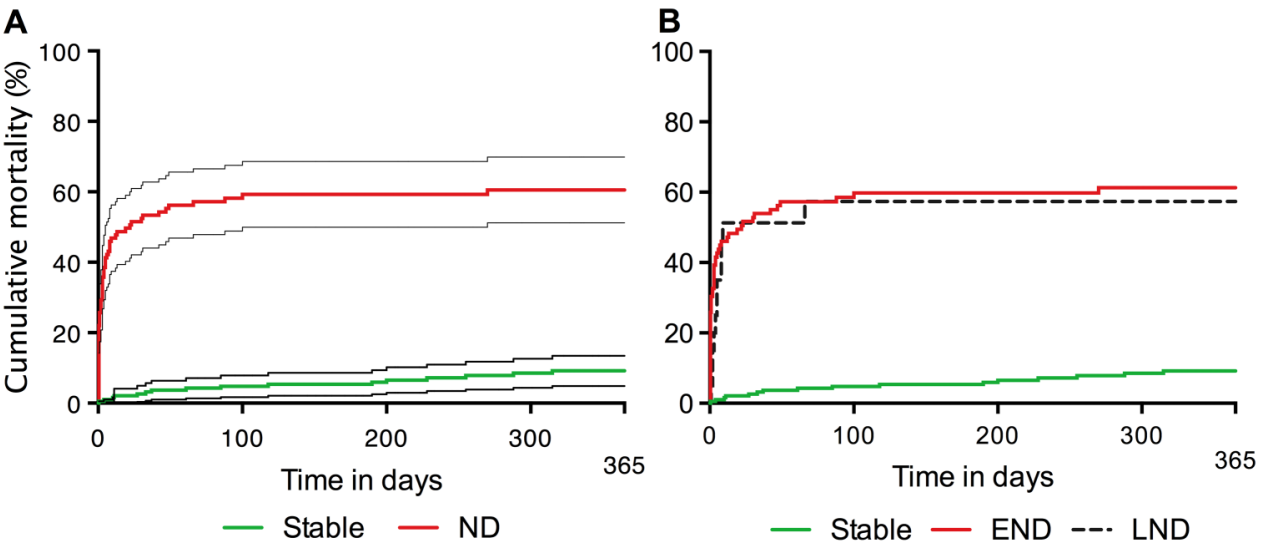

Figure 2 Survival curves displaying mortality during the first year. Panel $(\mathrm{A})$ shows the cumulative mortality $( \pm 95 \% \mathrm{Cl})$ of stable patients and patients with neurological deterioration (ND) during the first week. Panel (B) shows the cumulative mortality separated into patients with early ND (END), late ND (LND), and stable neurological status during the first week.

\section{DISCUSSION}

Patients who remained stable during the first week after stroke onset were most likely to survive the first year. Haematoma expansion is a key driving force behind END, and factors that mark the patients as unstable within the first day were essentially risk factors of haematoma expansion-spot sign, larger haematomas and anticoagulation treatment. Patients with smaller haematomas seemed to be protected from END. In addition, extensive haemorrhage into the ventricles of the brain marked the patients as unstable during the entire first week.

This study has several strengths. We believe that the generalisability of the data is high, as this study is based on consecutive patients with as little selection as possible as they were admitted based on the catchment area. Patients are admitted fast track with early symptoms of stroke, independent of, for example, age or stroke severity. The electronic follow-up of patients allowed this to be almost complete, and only very few (all foreign citizens) were lost to follow-up. The large majority of patients in this study underwent acute CTA enabling us to investigate the spot sign as a risk factor of ND.

Ovesen C, et al. BMJ Open 2015;5:e008563. doi:10.1136/bmjopen-2015-008563 
A weakness is the incomplete use of follow-up imaging, which could impose concerns of selection bias in the analysis of the follow-up images; however, the frequency of follow-up imaging was distributed equally among stable patients and patients suffering END or LND. It remains likely that the moribund patients, who might have concealed a large portion of haematoma expansion or IVH, as well as patients with mild symptoms were the groups most likely to be excluded from the follow-up scan. Another weakness is the way in which LND was assessed. We did not possess consecutive GCS and SIP score data further than the first day in most patients, and we thus needed to rely on medical chart data with documentation of GCS and the physicians' description of LND. However, physicians in Denmark are obligated to keep rigorous medical records, and because of this we believe that even though it could provide information bias, it was an acceptable way to assess LND.

Our data are consistent with other studies documenting a substantial risk of ND during the first 24-48 h. The relatively high fraction of patients undergoing END in our study is most likely due to the exclusion of comatose and surgically treated patients ${ }^{2-4}$ from other studies on END. LND is relatively poorly described in the literature. Mayer $e t a l^{4}$ documented an incidence of LND relatively similar to ours. Sun $e t a t^{6}$ found a higher incidence of LND, but did not exclude patients suffering END. We demonstrated that patients who already have suffered END will be at high risk of continuing their trajectory of deterioration during the following days and consequently give rise to a higher risk of LND if they are not excluded.

In a newly published meta-analysis, ${ }^{27}$ it was concluded that admission haematoma volume and IVH were associated with END; however, it was not shown that haematoma expansion was significantly related to END. Our study supports the notion that haematoma expansion is one of the key driving forces behind END. This study, as well as other studies, has found that patients with early predictors of haematoma expansion such as large haematoma, ${ }^{28}$ anticoagulation treatment and spot $\operatorname{sign}^{3}{ }^{19}$ were more likely to undergo END. In addition, a few studies have shown a direct link between haematoma expansion and END. ${ }^{10}{ }^{11}{ }^{29}$ In this study, we found the spot sign to be an important predictor of END. The spot sign is a well-recognised predictor of early haematoma expansion. It is likely that much of the effect of other predictors of END (larger haematomas and anticoagulation therapy) is mediated at least in part by the spot sign. This was also observed in this study, as some of the predictors of END lost predictive effect when the spot sign was considered in the model. This makes prediction of early neurological stability perhaps the most clinically useful utility of acute CTA, and spot sign assessment in patients with ICH should be recommended in all patients with acute ICH.

The consensus that patients with IVH are in risk of END seems to be well established. ${ }^{27}$ Our data further support Sun $e t a t^{6}$ in the link between LND and IVH. It is most likely that part of the process of both END and LND is driven by obstruction of the cerebrovascular fluid circulation and other harmful processes related to $\mathrm{IVH}^{7}{ }^{7} 30 \quad 31$ We encountered two phenomena not described in great detail in the literature-delayed IVH and expansion of the IVH volume. Both of these have previously been described as independent predictors of poor outcome-to the best of our knowledge never before in relation to $\mathrm{ND},{ }^{32} 33$ though intuitively correlated. Our study indicates that the final IVH volume is predictive of LND. This emphasises the need for follow-up imaging in all patients with ICH allowing earlier intervention.

It is clear from our results that the first week constitutes the primary critical period regarding functional outcome and long-term mortality. Even after we controlled for other well-known predictors of mortality and poor outcome ${ }^{26}$ as well as surgical treatment strategies, ND remained an important independent predictor of functional outcome and mortality. As the stability of the patients during the first week is very critical, when it comes to functional outcome and mortality, this study emphasises the importance of targeting the underlying processes that drive the deterioration-haematoma expansion, obstruction of circulation in the fluid-filled spaces, and comorbidities. It is our belief that this study possesses excellent external validity. The trend today is towards a more fast track workup of patients with stroke due to the documented benefit of recanalisation therapies in ischaemic stroke. This entails that more and more patients with ICH also will be diagnosed early. We believe that our fairly unselected population and the few patients lost to follow-up makes our study a trustworthy picture of ND in patients with acute ICH.

\section{CONCLUSION}

The first week constitutes a very critical period with a relative high incidence of ND. Stability during the first week entails a good prognosis. END seems to be directly associated with haematoma expansion. Obstruction of the fluid-filled spaces seems to be associated with both END and LND. Patients with a higher degree of comorbidity are at a higher risk of LND.

Contributors $\mathrm{CO}$ and $\mathrm{HC}$ contributed to the study concept and design. $\mathrm{CO}$, AFC, IH, CKH and EK participated in the data collection. CO, AFC, SR and HC participated in the analysis and interpretation of the data. $\mathrm{CO}$ drafted the manuscript. All the authors performed a critical revision of the manuscript for important intellectual content.

Funding This research received no specific grant from any funding agency in the public, commercial or not-for-profit sectors.

Competing interests None declared.

Ethics approval The Danish Data Protection Agency (file no. 2010-41-5205) has approved the registry.

Provenance and peer review Not commissioned; externally peer reviewed.

Data sharing statement No additional data are available. 
Open Access This is an Open Access article distributed in accordance with the Creative Commons Attribution Non Commercial (CC BY-NC 4.0) license, which permits others to distribute, remix, adapt, build upon this work noncommercially, and license their derivative works on different terms, provided the original work is properly cited and the use is non-commercial. See: http:// creativecommons.org/licenses/by-nc/4.0/

\section{REFERENCES}

1. Qureshi Al, Safdar K, Weil J, et al. Predictors of early deterioration and mortality in Black Americans with spontaneous intracerebral hemorrhage. Stroke 1995;26:1764-7.

2. Leira R, Davalos A, Silva $Y$, et al. Early neurologic deterioration in intracerebral hemorrhage: predictors and associated factors. Neurology 2004;63:461-7.

3. Rodriguez-Luna D, Rubiera M, Ribo M, et al. Ultraearly hematoma growth predicts poor outcome after acute intracerebral hemorrhage. Neurology 2011;77:1599-604.

4. Mayer SA, Sacco RL, Shi T, et al. Neurologic deterioration in noncomatose patients with supratentorial intracerebral hemorrhage. Neurology 1994:44:1379-84

5. Delgado P, Alvarez-Sabin J, Abilleira S, et al. Plasma d-dimer predicts poor outcome after acute intracerebral hemorrhage. Neurology 2006;67:94-8.

6. Sun W, Pan W, Kranz PG, et al. Predictors of late neurological deterioration after spontaneous intracerebral hemorrhage. Neurocrit Care 2013;19:299-305.

7. Hallevi H, Walker KC, Kasam M, et al. Inflammatory response to intraventricular hemorrhage: time course, magnitude and effect of t-PA. J Neurol Sci 2012;315:93-5.

8. Zazulia AR, Diringer MN, Derdeyn CP, et al. Progression of mass effect after intracerebral hemorrhage. Stroke 1999;30:1167-73.

9. Kiphuth IC, Huttner HB, Breuer L, et al. Sonographic monitoring of midline shift predicts outcome after intracerebral hemorrhage. Cerebrovasc Dis 2012;34:297-304.

10. Brott T, Broderick J, Kothari R, et al. Early hemorrhage growth in patients with intracerebral hemorrhage. Stroke 1997;28:1-5.

11. Ovesen C, Christensen AF, Krieger DW, et al. Time course of early postadmission hematoma expansion in spontaneous intracerebral hemorrhage. Stroke 2014;45:994-9.

12. Kazui S, Naritomi H, Yamamoto $\mathrm{H}$, et al. Enlargement of spontaneous intracerebral hemorrhage. Incidence and time course. Stroke 1996;27:1783-7.

13. Langhorne P, Fearon P, Ronning OM, et al. Stroke unit care benefits patients with intracerebral hemorrhage: systematic review and meta-analysis. Stroke 2013;44:3044-9.

14. Evans A, Perez I, Harraf F, et al. Can differences in management processes explain different outcomes between stroke unit and stroke-team care? Lancet 2001;358:1586-92.

15. Steiner T, Kaste M, Forsting M, et al. Recommendations for the management of intracranial haemorrhage-part l: spontaneous intracerebral haemorrhage. The European Stroke Initiative Writing Committee and the Writing Committee for the EUSI Executive Committee. Cerebrovasc Dis 2006;22:294-316.

16. Steiner T, Al-Shahi Salman R, Beer R, et al. European Stroke Organisation (ESO) guidelines for the management of spontaneous intracerebral hemorrhage. Int J Stroke 2014;9:840-55.
17. Jorgensen HS, Nakayama $\mathrm{H}$, Raaschou $\mathrm{HO}$, et al. Effect of blood pressure and diabetes on stroke in progression. Lancet 1994;344:156-9.

18. Fan JS, Chen $\mathrm{YC}$, Huang $\mathrm{HH}$, et al. The association between on-scene blood pressure and early neurological deterioration in patients with spontaneous intracerebral haemorrhage. Emerg Med $J$ 2015;32:239-43.

19. Demchuk AM, Dowlatshahi D, Rodriguez-Luna D, et al. Prediction of haematoma growth and outcome in patients with intracerebral haemorrhage using the CT-angiography spot sign (predict): a prospective observational study. Lancet Neurol 2012;11: 307-14.

20. Gray LJ, Ali M, Lyden PD, et al. Virtual International Stroke Trials Archive Collaboration. Interconversion of the National Institutes of Health Stroke Scale and Scandinavian Stroke Scale in acute stroke. $J$ Stroke Cerebrovasc Dis 2009;18:466-8.

21. Sorimachi T, Fujii Y. Early neurological change in patients with spontaneous supratentorial intracerebral hemorrhage. J Clin Neurosci 2010;17:1367-71.

22. Charlson ME, Pompei $\mathrm{P}$, Ales $\mathrm{KL}$, et al. A new method of classifying prognostic comorbidity in longitudinal studies: development and validation. J Chronic Dis 1987;40:373-83.

23. Wada R, Aviv RI, Fox AJ, et al. CT angiography "spot sign" predicts hematoma expansion in acute intracerebral hemorrhage. Stroke 2007;38:1257-62.

24. Kothari RU, Brott T, Broderick JP, et al. The ABCs of measuring intracerebral hemorrhage volumes. Stroke 1996;27:1304-5.

25. Graeb DA, Robertson WD, Lapointe JS, et al. Computed tomographic diagnosis of intraventricular hemorrhage. Etiology and prognosis. Radiology 1982;143:91-6.

26. Poon MT, Fonville AF, Al-Shahi Salman R. Long-term prognosis after intracerebral haemorrhage: systematic review and meta-analysis. J Neurol Neurosurg Psychiatry 2014;85: 660-7.

27. Specogna AV, Turin TC, Patten SB, et al. Factors associated with early deterioration after spontaneous intracerebral hemorrhage: a systematic review and meta-analysis. PLOS ONE 2014; 9:e96743.

28. Dowlatshahi D, Smith EE, Flaherty ML, et al. Small intracerebral haemorrhages are associated with less haematoma expansion and better outcomes. Int J Stroke 2011;6:201-6.

29. Lord AS, Gilmore E, Choi HA et al. VISTA-ICH Collaboration. Time course and predictors of neurological deterioration after intracerebral hemorrhage. Stroke 2015;46:647-52.

30. Bhattathiri PS, Gregson B, Prasad KS, et al. Intraventricula hemorrhage and hydrocephalus after spontaneous intracerebral hemorrhage: results from the STICH trial. Acta Neurochir Suppl 2006;96:65-8

31. Tuhrim S, Horowitz DR, Sacher M, et al. Volume of ventricular blood is an important determinant of outcome in supratentorial intracerebral hemorrhage. Crit Care Med 1999;27:617-21.

32. Maas MB, Nemeth AJ, Rosenberg NF, et al. Delayed intraventricular hemorrhage is common and worsens outcomes in intracerebral hemorrhage. Neurology 2013;80:1295-9.

33. Steiner T, Diringer MN, Schneider D, et al. Dynamics of intraventricular hemorrhage in patients with spontaneous intracerebral hemorrhage: risk factors, clinical impact, and effect of hemostatic therapy with recombinant activated factor VII. Neurosurgery 2006;59:767-73; discussion 773-4. 